\title{
Nonlinear Absorption of Ag Nanoparticles coupled the Local Light Field in the Slit of ZnO microrod
}

\author{
$\mathrm{Ru}$ Wang, Chuanxiang $\mathrm{Xu}$ \\ State Key Laboratory of Bioelectronics, School of Physics, Southeast University, Nanjing 210096, China
}

Lasing mode regulation is essential for realizing the practical applications such as optical communication and optical sensing/switches. In this work, we etched the slit along the c-axis of a hexagonal $\mathrm{ZnO}$ microrod by focusing ion beam etching. This slit can produce very strong local light field under strong incident light. Compared with the bare $\mathrm{ZnO}$ sample, WGM lasing mode is reduced, while the emission intensity decreases and the laser threshold increases. Then Ag nanoparticles filled the slit as nonlinear absorbers. the regulation of WGM laser mode is realized by the coupling effect of non-linear absorption effect of Ag nano-particles and the local light field. Moreover, laser mode intensity is enhanced by strong coupling between $\mathrm{ZnO}$ microrod and surface plasmon resonant of Ag NPs of its surface that is proved by PL decay time.

\section{Results and discussion}
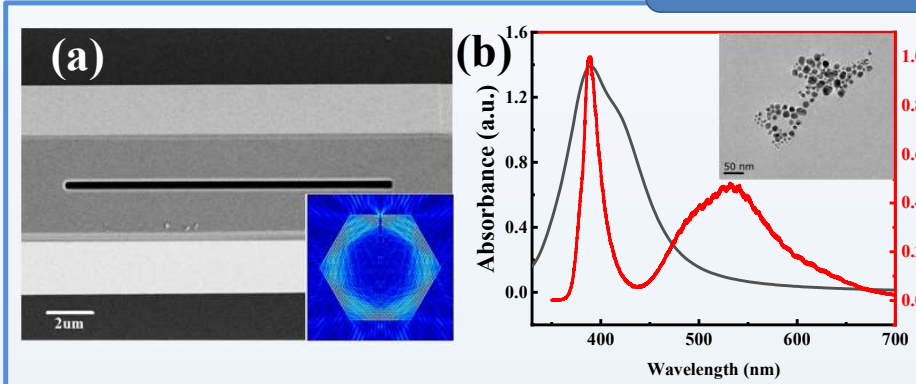

Figure. 1 (a) SEM images of $\mathrm{ZnO}$ microrod with the slit, Inset is the far field emission pattern of ZnO-Slit sample; (b) The PL spectrum of ZnO-Slit (red line) and the absorption spectrum of Ag NPs (black line), inset is TEM image of Ag NPs.


Figure. 2 (a) Laser mode spectra of $\mathrm{ZnO}$-Bare and $\mathrm{ZnO}$-Slit with $141 \mu \mathrm{W}$ excitation power; (b) The dependence of the emission intensities on different excitation powers for $\mathrm{ZnO}$-Bare and $\mathrm{ZnO}$-Slit
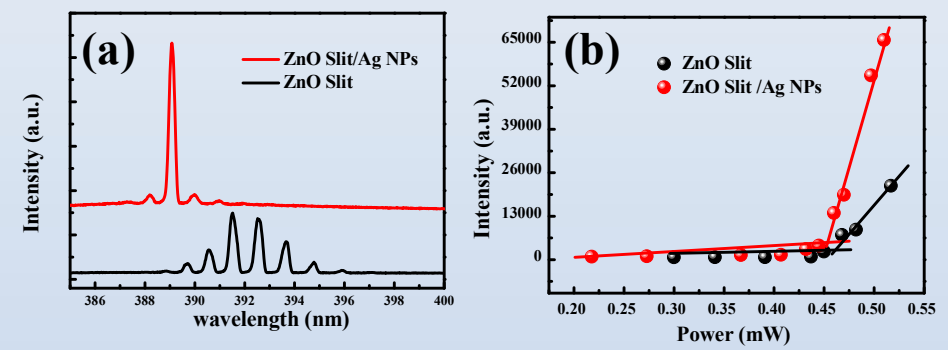



Figure. 3 (a) Laser mode spectra of ZnO-Slit before and after Ag NPs decoration at $141 \mu \mathrm{W}$ excitation power. Dependence of (b) the emission intensity, (c)full width at half maximum on the pumping power for $\mathrm{ZnO}$ Slit before and after Ag NPs decoration; (d) electric field enhancement image of Ag nanoparticles on the surface of $\mathrm{ZnO}$-Slit/Ag NPs sample.

(a)
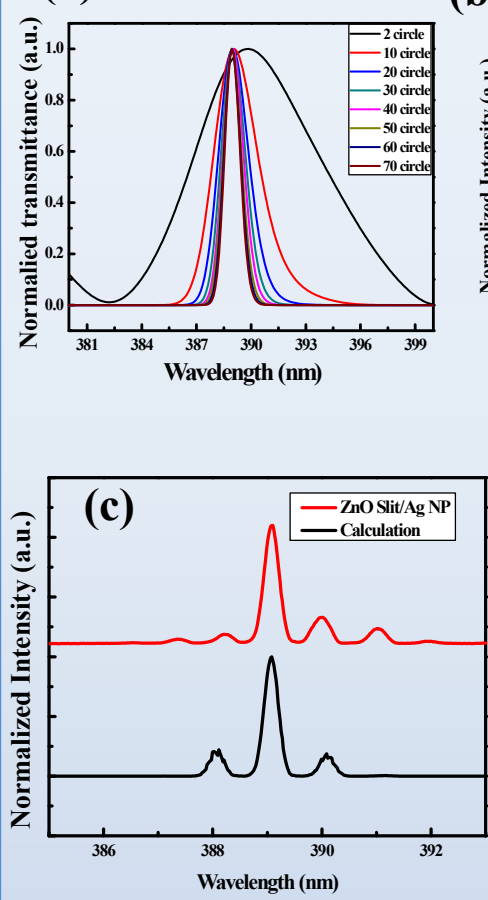

(b)



Figure. 4 (a) The transmission spectrum of light passing through Ag nanoparticles with different cycle in the slit and (b) the red line is the laser spectrum of passive cavity, the black line is the gain spectrum of $\mathrm{ZnO}$ microrod with slit, and the blue line is the transmission spectrum of Ag nanoparticles passing through the slit for 40 times.(c) The WGM laser spectrum obtained by using the convolution of the three curves in Figure (b) and the measured power of $0.493 \mathrm{~mW} \mathrm{ZnO}$ slit microrod laser spectrum after the addition of $\mathrm{Ag}$ nanoparticles.

\section{Reference}

[1] Hossein Hodaei, et al. Science 2014, 346(6212), 975-978

[2] Pan Wang,et al. Nano Lett. 2015, 15, 7581-7586 [3] Gangyi Zhu et al, ACS Appl. Mater. Interfaces 2012, 4, 6195-6201 2. Ag nanoparticles filled in the ZnO slit can act as an absorbing filter.

[4]X. He et al. Applied Surface Science 283 (2013) 571-576

[5] Zhiqiang Mu, Nano Lett 17(2017), 1552-1558

\section{conclusion}

1. Ag nanoparticles can enhance the laser intensity of $\mathrm{ZnO}$ WGM microcavity.

3. The $\mathrm{ZnO} / \mathrm{AgWGM}$ microcavity can control the laser mode. 